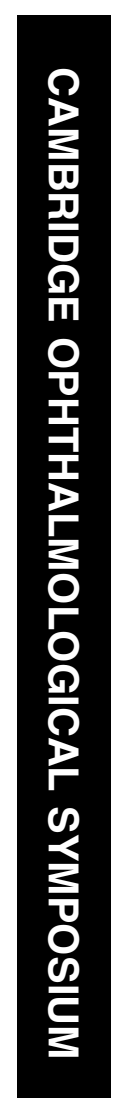

Doheny Image Reading Center, Doheny Eye Institute, Keck School of Medicine of the University of Southern California, Los Angeles, CA, USA

Correspondence: SR Sadda, Doheny Image Reading Center,

Doheny Eye Institute, DEI 3623,

1450 San Pablo Street, Los Angeles,

CA 90033,

USA

Tel: + 1323442 6503;

Fax: +1 323442 6460;

E-mail: ssadda@doheny.org

Received: 13 October 2009 Accepted in revised form:

17 November 2009

Published online:

11 December 2009

Presented at the 39th

Cambridge

Ophthalmological

Association Symposium

\section{Imaging chorioretinal vascular disease}

PA Keane and SR Sadda

\section{Introduction}

In 1961, Novotny and Alvis ${ }^{1}$ produced the first fluorescein angiograms providing images of the chorioretinal vascular system. Since that time, chorioretinal imaging - principally stereoscopic photography and fluorescein angiography - had a crucial role in the management of patients with chorioretinal vascular diseases such as neovascular agerelated macular degeneration (AMD). More recently, the development of a new imaging modality, optical coherence tomography (OCT), has addressed many of the limitations of these traditional imaging techniques, and reinforced the central role of imaging in the management of these patients. In this article, we describe the principal chorioretinal imaging techniques in use today, as well as a number of new technologies currently in development that may transform the management of chorioretinal vascular disease.

\section{Fluorescein angiography}

Fluorescein angiography possesses a number of features that have made it central to the management of chorioretinal vascular disease. ${ }^{2}$ In particular, it permits visualization of the retinal microcirculation in exquisite detail, allowing identification of vascular abnormalities and areas of retinal nonperfusion. It also enables visualization of many pathologic changes affecting the choroidal vasculature (Figure 1). Through the assessment of vascular leakage, fluorescein angiography also provides important functional information (ie the integrity of the blood-retinal barrier). These features have allowed fluorescein angiography to show its worth in a wide variety of clinical settings, as well as in the context of important randomized clinical trials (eg the Early Treatment of Diabetic Retinopathy Study (ETDRS) for diabetic retinopathy). ${ }^{3}$ 


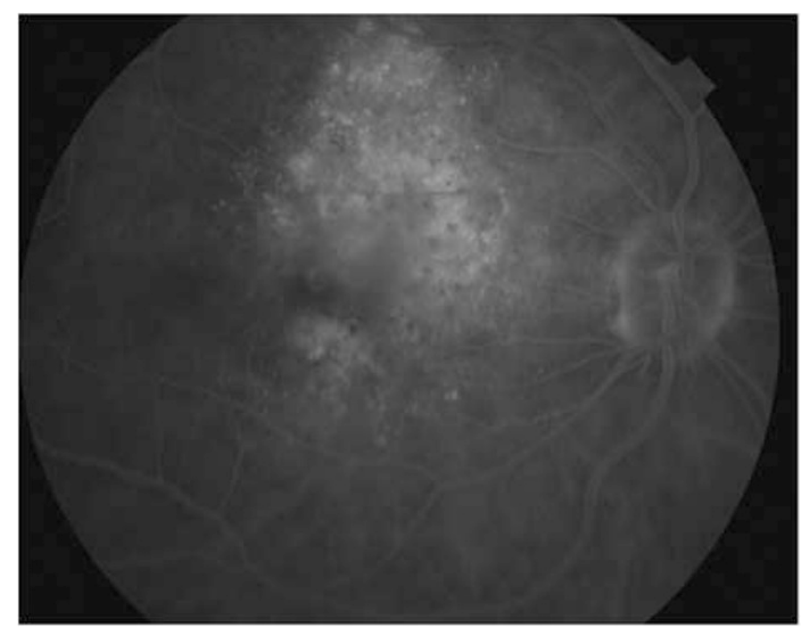

Figure 1 Fluorescein angiogram showing evidence of late stippled hyperfluorescence consistent with fibrovascular pigment epithelium detachment.

\section{Limitations of fluorescein angiography}

Despite these advantages, fluorescein angiography has a number of limitations. First, it is an invasive procedure with complications that range from minor (nausea and vomiting) to serious (anaphylaxis). Second, its ability to visualize the choroidal circulation is limited, with considerable loss of vascular detail over time because of progressive dye leakage. Third, even with the use of stereoscopic images, it has a limited axial resolution that allows - at best - semi-quantitative measurement of retinal thickness that is subject to considerable variability. ${ }^{4}$ Finally, fluorescein angiography provides only limited information regarding the structural and functional consequences of vascular disease. Fortunately, in recent years, advances in other chorioretinal imaging modalities have addressed many of these deficiencies.

\section{Indocyanine green angiography}

Indocyanine green angiography, first described in 1972, allows enhanced visualization of the choroidal circulation - unlike fluorescein, indocyanine green is almost completely bound to protein and tends not to leak through the fenestrated capillaries of the choriocapillaris obscuring the larger choroidal vessels (Figure 2). ${ }^{5}$ Despite this, initial usage was limited by the poor fluorescence efficiency of indocyanine green, and the limited ability to produce high-resolution images on infrared film. Since the 1990s, however, the development of high-speed, high-resolution digital imaging systems has resolved many of these issues. ${ }^{6}$ Although still not commonly performed in clinical practice, indocyanine green angiography has improved our understanding of disorders such as neovascular AMD (especially retinal angiomatous proliferation and polypoidal choroidal

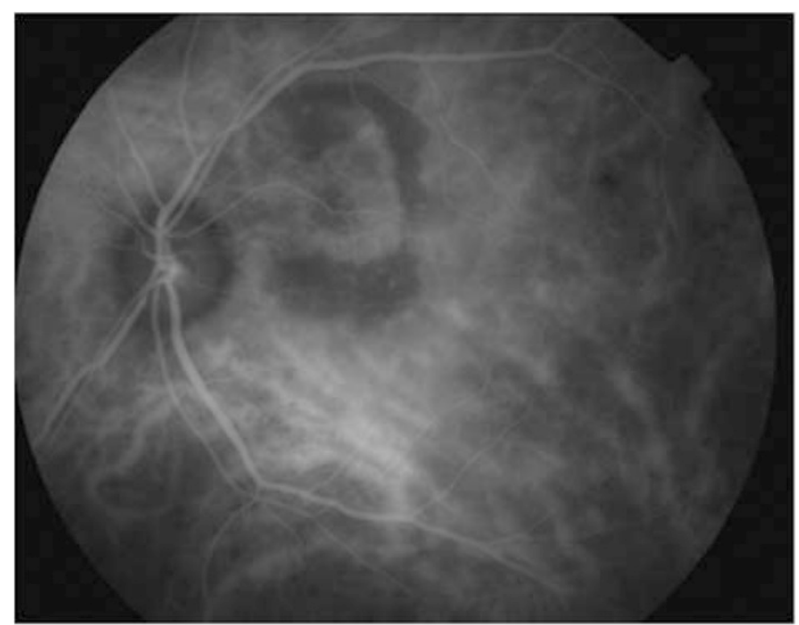

Figure 2 Indocyanine green angiography showing evidence of peripapillary choroidal neovascularization.

vasculopathy), central serous chorioretinopathy, and chorioretinal inflammatory disorders. ${ }^{7}$

\section{Fundus autofluorescence}

Autofluorescence is an intrinsic property of many structures within the eye (eg retinal pigment epithelium (RPE)), such that transient emission of light occurs when these structures are illuminated by an exogenous source. ${ }^{8}$ Autofluorescence of the RPE is related to the intracellular accumulation of lipofuscin, a byproduct of incomplete photoreceptor outer segment degradation. Lipofuscin accumulation is a hallmark of normal aging in many cells, but may also be a common downstream pathogenic mechanism in a number of retinal degenerative diseases. As a result, fundus autofluorescence (FAF) imaging has generated considerable interest in recent years for patients with both inherited and acquired retinal degenerations (Figure 3). In fact, FAF imaging has recently been approved by the US Food and Drug Administration as a primary end point in clinical trials of nonneovascular AMD (geographic atrophy). ${ }^{9}$

The role of FAF imaging in chorioretinal vascular disease is less well established. In neovascular AMD, for example, the continuity of the FAF signal over the lesion may provide prognostic information. ${ }^{10}$ In patients with this disorder, FAF may be relatively normal ('continuous') early on, a finding that correlates with better visual acuity, and presumably represents continued RPE viability. However, with longer-standing disease, decreased FAF is often seen, a finding that correlates with decreased visual acuity, and presumably represents photoreceptor loss and RPE atrophy. FAF imaging may also be of diagnostic use in less commonly 


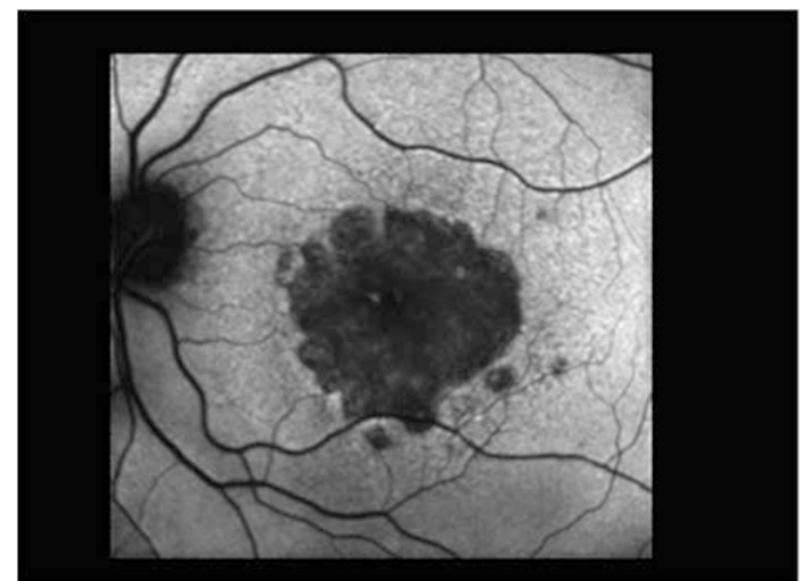

Figure 3 Fundus autofluorescence image obtained with a confocal scanning laser ophthalmoscope-geographic atrophy may be clearly seen as a central area of hypoautofluorescence.

seen retinal vascular disorders such as type 2 idiopathic juxtafoveal telangiectasia, in which central depletion of macular pigment results in a characteristic loss of normal foveal hypofluorescence. ${ }^{11}$

\section{Optical coherence tomography}

OCT, first described in 1991, allows high-resolution cross-sectional images of the neurosensory retina to be obtained in a non-invasive manner. ${ }^{12}$ As a result, retinal imaging with OCT has quickly become an integral tool for the management of chorioretinal vascular disorders.

\section{Time domain OCT}

OCT works by measuring the properties of light waves reflected from tissue (analogous to ultrasonography). ${ }^{13}$ In the original OCT systems, movement of a reference mirror allowed acquisition of depth information over time - 'time domain' OCT. The release of the first commercial time domain OCT systems, in 1996 (OCT1) and 2000 (OCT2), quickly established the clinical benefits of OCT - in particular for the depiction of the vitreomacular interface. However, it was the release of the third generation of time domain OCT devices in 2002 that heralded the widespread adoption of OCT by retinal specialists. OCT3 (Stratus OCT, Carl Zeiss Meditec, Dublin, CA, USA) offered faster scanning speed (400 Ascans per second) and higher resolution (8-10 microns axially) - features that provided significant advantages for the management of chorioretinal vascular diseases.

\section{Clinical applications of time domain OCT}

Macular oedema is a common cause of vision loss in patients with retinal vascular disease. ${ }^{14}$ The greater axial resolution of Stratus OCT (particularly when compared to stereoscopic fundus photography) has allowed improved characterization of the structural changes that occur in macular oedema. In diabetic macular oedema, for example, distinct patterns of morphologic change may be seen on OCT: diffuse retinal thickening, cystoid macular oedema, serous retinal detachment, and vitreomacular traction (retinal vascular diseases are also commonly accompanied by epiretinal membrane formation) (Figure 4). ${ }^{15-17}$ In addition to these qualitative assessments, measurements of retinal thickness provided by time domain OCT have become important criteria for determining eligibility for clinical trials, as well as being adopted as anatomic end points in these trials. ${ }^{18}$

In recent years, driven by the seminal findings of the PrONTO study for neovascular AMD, OCT has also been rapidly adopted for the management of patients with choroidal vascular disease. ${ }^{19,20}$ In the PrONTO study, OCTderived criteria were used both for determination of eligibility, and for re-treatment decisions (eg presence of subretinal fluid), in patients receiving intravitreal ranibizumab. In 2007, the following OCT-derived guidelines, for the treatment of neovascular AMD, were suggested by Brown and Regillo: (1) initial monthly treatment, until no intraretinal, subretinal, or sub-RPE fluid; (2) consideration of fluorescein angiography if visual acuity changes do not correlate with anatomic improvements; (3) re-treatment based on qualitative inspection of all six high-resolution Stratus OCT scans with re-treatment for any recurrence of intraretinal, subretinal, or sub-RPE fluid. ${ }^{21}$ These guidelines or variations, thereof, have been quickly adopted by retina specialists worldwide, greatly increasing the utilization of OCT in the management of patients with choroidal vascular disease.

\section{Limitations of time domain OCT}

Although it is clear that OCT successfully addresses many of the limitations of fluorescein angiography, the use of OCT in choroidal vascular diseases has also highlighted many of the limitations of time domain OCT. OCT-derived retinal thickness values are obtained by automated detection (segmentation) of the inner and outer retinal boundaries. Unfortunately, however, this automated detection frequently fails in patients with retinal disease, particularly in patients with choroidal vascular disease. ${ }^{22}$ Moreover, even if boundary detection is correct, many specific disease components are not quantified by OCT (eg subretinal fluid, pigment epithelium detachments). To ensure the accuracy of retinal thickness measurements, and to allow quantification of other morphologic parameters, manual segmentation of OCT images is often performed in dedicated image reading centres. ${ }^{23-25}$ 

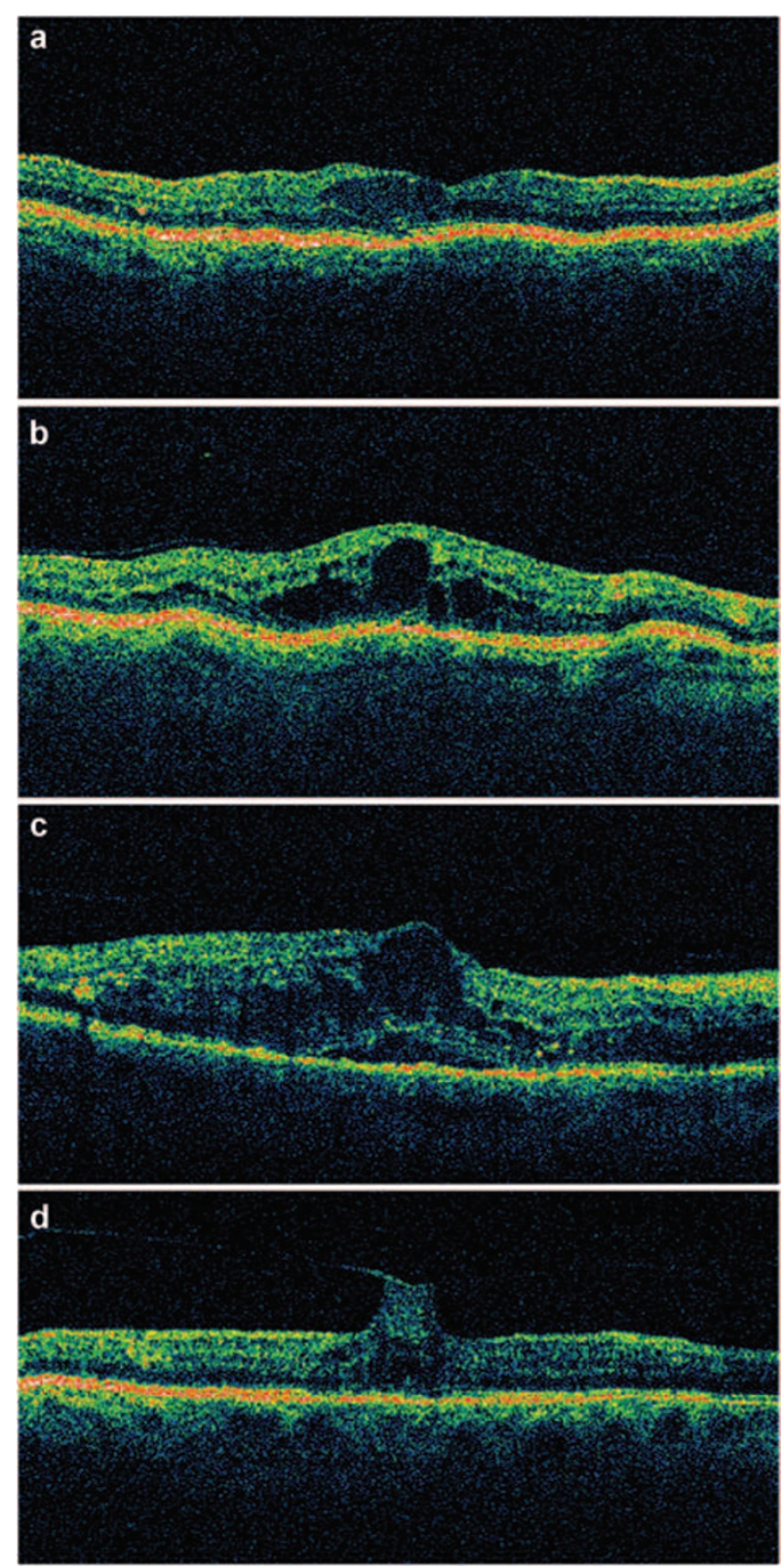

Figure 4 Patterns of structural change on optical coherence tomography (OCT) in patients with diabetic macular oedema. OCT B-scans show sponge-like retinal thickening (a), cystoid macular oedema (b), serous retinal detachment (c), and vitreomacular traction with peaking of the retinal surface (d).

Time domain OCT systems are also limited by their requirement for a mobile reference mirror - a requirement that limits their image acquisition speed. ${ }^{26}$ Consequently, typical time domain OCT scanning protocols capture $<5 \%$ of the macula in a single image set and significant interpolation is required to construct retinal thickness maps. As a result, time domain OCT scanning protocols often miss small lesions that fall between the scanned lines, and any segmentation errors that occur may often be propagated across large areas. Fortunately, these limitations have been largely overcome in recent years with the latest generation of commercial OCT technology-'spectral domain' OCT.

\section{Spectral domain $O C T$}

In spectral domain OCT systems, the use of spectral interferometry and a mathematical function (Fourier transformation) removes the need for a mobile reference mirror, and allows images to be acquired 50-100 times more quickly than in time domain systems (typically over 20000 A-scans per second). ${ }^{27-29}$ The high speed of spectral domain OCT allows significantly greater coverage of the macular area using raster scanning protocols (eg 128 B-scans, with each B-scan consisting of 512 A-scans). The greater speed of spectral domain OCT also reduces the frequency of eye motion artefacts and allows the creation of three-dimensional reconstructions of the retina. In addition, by summing the intensity values in each A-scan, the dense scanning of spectral domain OCT allows generation of 'projection' images that appear superficially similar to fundus photographic images. These projection images contain invariant landmarks that can be aligned with standard fundus photographic images, facilitating direct comparison with these images and allowing more precise registration for inter-visit comparisons. ${ }^{30}$

The rapid scanning of spectral domain OCT also allows averaging of multiple B-scan images to be readily performed, reducing speckle noise and allowing greater visualization of fine structures - in particular, the structures of the outer retina and choroid. ${ }^{31}$ Although spectral domain OCT has a higher sensitivity than time domain OCT, spectral domain image quality changes as the scan moves vertically on the screen. By adjusting the spectral domain OCT device to maximize its sensitivity at the choroid, and through the use of B-scan averaging, extremely high-quality images may be obtained. Such imaging allows clear visualization of outer retinal structures such as the external limiting membrane, and enhanced visualization of the architecture of fibrovascular tissue in neovascular AMD (Figure 5). ${ }^{32-34}$

\section{Current limitations and future directions}

Although it represents a significant advance, spectral domain OCT also has a number of limitations. As with time domain OCT, the transverse resolution of spectral domain OCT is limited by the optics of the ocular system and, as a result, spectral domain OCT does not allow visualization of individual cells. ${ }^{35}$ In addition, the functional data provided by spectral domain OCT 

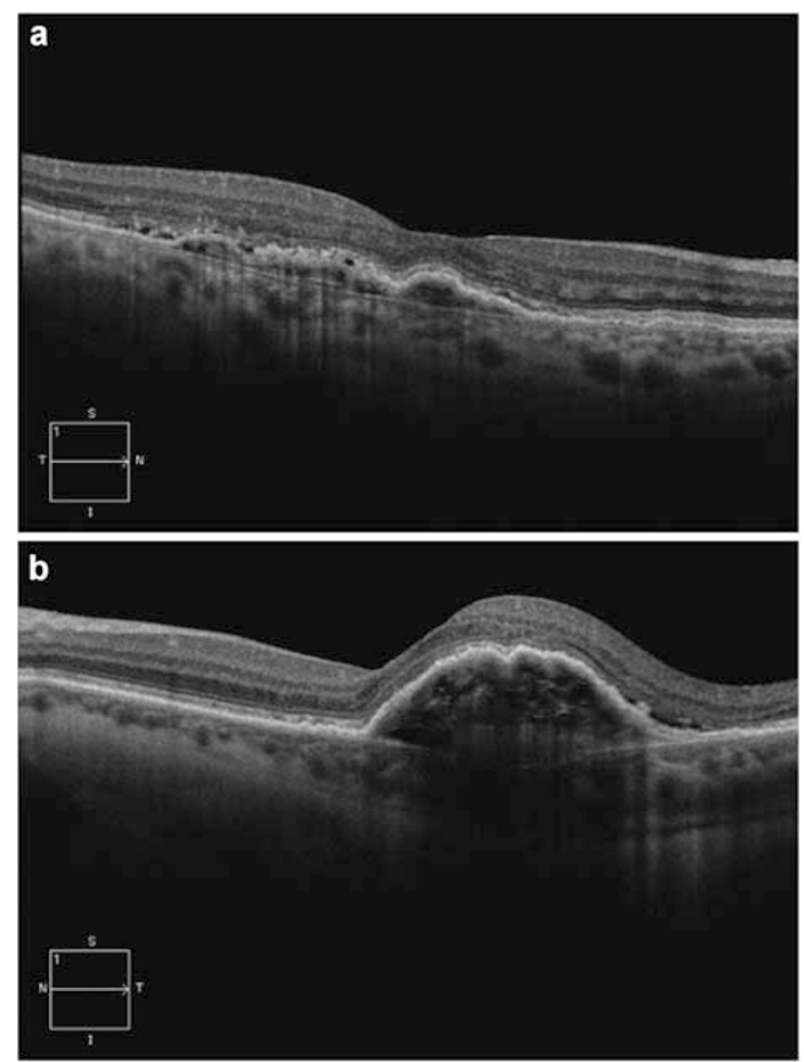

Figure 5 Evaluation of neovascular age-related macular degeneration (AMD) using 'enhanced depth' spectral domain optical coherence tomography (Cirrus HD-OCT, Carl Zeiss Meditec, Dublin, CA, USA) - (a) and (b).

remains rudimentary at best. Furthermore, better segmentation software is still required to facilitate precise quantitative subanalysis of dense raster scan data sets. ${ }^{36}$ Not surprisingly, future OCT technologies will likely address these limitations. Prototype Doppler OCT systems allow measurement of retinal blood flow by the assessment of light reflectivity changes in retinal blood vessels over short time periods. ${ }^{37}$ 'Swept-source' OCT systems allow significant increases in imaging sensitivity and speed (eg >300000 A-scans per second), through the use of a tunable laser, ${ }^{38}$ whereas polarization-sensitive OCT may prove to encode much of the information provided by FAF. ${ }^{39}$ Finally, the use of adaptive optics in OCT devices may increase the transverse resolution of OCT systems and provide cellular level detail. ${ }^{40}$

\section{Conclusion}

In recent years, retinal imaging with OCT has become central to the treatment of patients with chorioretinal disorders. Using current, commercially available, technology, it is now possible to obtain high-quality cross-sectional images of the choroid - such imaging may represent the next frontier in our understanding of retinal disease pathogenesis. Furthermore, the applications of OCT in chorioretinal vascular disease are likely to grow with functional extensions of this technology in the near future (eg Doppler OCT may lead to a new wave of categorizing retinal vascular disorders on the basis of blood flow). Such advances, in association with improvements in other imaging techniques, are likely to dramatically enhance our management of patients with chorioretinal disease.

\section{Conflict of interest}

Dr Sadda is a co-inventor of Doheny intellectual property related to optical coherence tomography that has been licensed by Topcon Medical Systems, and is a member of the scientific advisory board for Heidelberg Engineering. The Doheny Image Reading Center also receives research support from Carl Zeiss Meditec and Optovue Inc.

\section{Acknowledgements}

This work is supported in part by NIH Grant EY03040 and NEI Grant R01 EY014375.

\section{References}

1 Novotny HR, Alvis DL. A method of photographing fluorescence in circulating blood in the human retina. Circulation 1961; 24: 82-86.

2 Yannuzzi LA, Ober MD, Slakter JS, Spaide RF, Fisher YL, Flower RW et al. Ophthalmic fundus imaging: today and beyond. Am J Ophthalmol 2004; 137: 511-524.

3 Early Treatment Diabetic Retinopathy Study Research Group. Photocoagulation for diabetic macular edema. Early Treatment Diabetic Retinopathy Study Report Number 1. Arch Ophthalmol 1985; 103: 1796-1806.

4 Kaiser RS, Berger JW, Williams GA, Tolentino MJ, Maguire $\mathrm{AM}$, Alexander $\mathrm{J}$ et al. Variability in fluorescein angiography interpretation for photodynamic therapy in age-related macular degeneration. Retina 2002; 22: 683-690.

5 Flower RW, Hochheimer BF. Clinical infrared absorption angiography of the choroid. Am J Ophthalmol 1972; 73: 458-459.

6 Yannuzzi LA, Slakter JS, Sorenson JA, Guyer DR, Orlock DA. Digital indocyanine green videoangiography and choroidal neovascularization. Retina 1992; 12: 191-223.

7 Stanga PE, Lim JI, Hamilton P. Indocyanine green angiography in chorioretinal diseases: indications and interpretation: an evidence-based update. Ophthalmology 2003; 110: 15-21.

8 Schmitz-Valckenberg S, Holz FG, Bird AC, Spaide RF. Fundus autofluorescence imaging: review and perspectives. Retina 2008; 28: 385-409. 
9 Schmitz-Valckenberg S, Fleckenstein M, Scholl HPN, Holz FG. Fundus autofluorescence and progression of age-related macular degeneration. Survey Ophthalmol 2009; 54: 96-117.

10 Vaclavik V, Vujosevic S, Dandekar SS, Bunce C, Peto T, Bird AC. Autofluorescence imaging in age-related macular degeneration complicated by choroidal neovascularization. A prospective study. Ophthalmology 2008; 115: 342-346.

11 Helb HM, Charbel Issa P, Van Der Veen RLP, Berendschot TTJM, Scholl HPN, Holz FG. Abnormal macular pigment distribution in type 2 idiopathic macular telangiectasia. Retina 2008; 28: 808-816.

12 Huang D, Swanson E, Lin C, Schuman J, Stinson W, Chang W et al. Optical coherence tomography. Science 1991; 254: 1178-1181.

13 Drexler W, Fujimoto JG. State-of-the-art retinal optical coherence tomography. Prog Retin Eye Res 2008; 27: 45-88.

14 Johnson MW. Etiology and treatment of macular edema. Am J Ophthalmol 2008; 147: 11-21.e1.

15 Keane PA, Sadda SR. Optical coherence tomography in the diagnosis and management of diabetic retinopathy. Int Ophthalmol Clin 2009; 49: 61-74.

16 Kim BY, Smith SD, Kaiser PK. Optical coherence tomographic patterns of diabetic macular edema. Am J Ophthalmol 2006; 142: 405-412.

17 Otani T, Kishi S, Maruyama Y. Patterns of diabetic macular edema with optical coherence tomography. Am J Ophthalmol 1999; 127: 688-693.

18 Diabetic Retinopathy Clinical Research Network. Relationship between optical coherence tomographymeasured central retinal thickness and visual acuity in diabetic macular edema. Ophthalmology 2007; 114: 525-536.

19 Fung AE, Lalwani GA, Rosenfeld PJ, Dubovy SR, Michels S Feuer WJ et al. An optical coherence tomography-guided, variable dosing regimen with intravitreal ranibizumab (Lucentis) for neovascular age-related macular degeneration. Am J Ophthalmol 2007; 143: 566-583.

20 Lalwani G, Rosenfeld P, Fung A, Dubovy S, Michels S, Feuer $\mathrm{W}$ et al. A variable-dosing regimen with intravitreal ranibizumab for neovascular age-related macular degeneration: Year 2 of the PrONTO study. Am J Ophthalmol 2009; 148: 43-58.e1.

21 Brown DM, Regillo CD. Anti-VEGF agents in the treatment of neovascular age-related macular degeneration: applying clinical trial results to the treatment of everyday patients. Am J Ophthalmol 2007; 144: 627-637.

22 Sadda SR, Wu Z, Walsh AC, Richine L, Dougall J, Cortez R et al. Errors in retinal thickness measurements obtained by optical coherence tomography. Ophthalmology 2006; 113: 285-293.

23 Keane PA, Chang KT, Liakopoulos S, Jivrajka RV, Walsh AC, Sadda SR. Effect of ranibizumab retreatment frequency on neurosensory retinal volume in neovascular AMD. Retina 2009; 29: 592-600.

24 Keane PA, Liakopoulos S, Chang KT, Wang M, Dustin L, Walsh AC et al. Relationship between optical coherence tomography retinal parameters and visual acuity in neovascular age-related macular degeneration. Ophthalmology 2008; 115: 2206-2214.

25 Keane PA, Liakopoulos S, Jivrajka RV, Chang KT, Alasil T, Walsh AC et al. Evaluation of optical coherence tomography retinal thickness parameters for use in clinical trials for neovascular age-related macular degeneration. Invest Ophthalmol Vis Sci 2009; 50: 3378-3385.
26 Keane PA, Bhatti RA, Brubaker JW, Liakopoulos S, Sadda SR, Walsh AC. Comparison of clinically relevant findings from high-speed Fourier-domain and conventional timedomain optical coherence tomography. Am J Ophthalmol 2009; 148: 242-248.e1.

27 Schmidt-Erfurth U, Leitgeb RA, Michels S, Povazay B, Sacu S, Hermann B et al. Three-dimensional ultrahighresolution optical coherence tomography of macular diseases. Invest Ophthalmol Vis Sci 2005; 46: 3393-3402.

28 Wojtkowski M, Bajraszewski T, czyñska I, Targowski P, Kowalczyk A, Wasilewski W et al. Ophthalmic imaging by spectral optical coherence tomography. Am J Ophthalmol 2004; 138: 412-419.

29 Wojtkowski M, Srinivasan V, Fujimoto J, Ko T, Schuman J, Kowalczyk A et al. Three-dimensional retinal imaging with high-speed ultrahigh-resolution optical coherence tomography. Ophthalmology 2005; 112: 1734-1746.

30 Gorczynska I, Srinivasan VJ, Vuong LN, Chen RWS, Liu JJ, Reichel E et al. Projection OCT fundus imaging for visualising outer retinal pathology in non-exudative agerelated macular degeneration. Br J Ophthalmol 2009; 93: 603-609.

31 Sakamoto A, Hangai M, Yoshimura N. Spectral-domain optical coherence tomography with multiple B-scan averaging for enhanced imaging of retinal diseases. Ophthalmology 2008; 115: 1071-1078.e7.

32 Fujiwara T, Imamura Y, Margolis R, Slakter JS, Spaide RF. Enhanced depth imaging optical coherence tomography of the choroid in highly myopic eyes. Am J Ophthalmol 2009; 148: 445-450.

33 Spaide RF. Enhanced depth imaging optical coherence tomography of retinal pigment epithelial detachment in age-related macular degeneration. Am J Ophthalmol 2009; 147: 644-652.

34 Spaide RF, Koizumi H, Pozonni MC. Enhanced depth imaging spectral-domain optical coherence tomography. Am J Ophthalmol 2008; 146: 496-500.

35 Drexler W. Cellular and functional optical coherence tomography of the human retina: the Cogan lecture. Invest Ophthalmol Vis Sci 2007; 48: 5339-5351.

36 Keane PA, Mand PS, Liakopoulos S, Walsh AC, Sadda SR. Accuracy of retinal thickness measurements obtained with cirrus optical coherence tomography. Br J Ophthalmol 2009; 93(11): 1461-1467.

37 Wang Y, Lu A, Gil-Flamer J, Tan O, Izatt JA, Huang D. Measurement of total blood flow in the normal human retina using Doppler Fourier-domain optical coherence tomography. Br J Ophthalmol 2009; 93: 634-637.

38 Srinivasan V, Adler D, Chen Y, Gorczynska I, Huber R, Duker $\mathrm{J}$ et al. Ultrahigh-speed optical coherence tomography for three-dimensional and en face imaging of the retina and optic nerve head. Invest Ophthalmol Vis Sci 2008; 49: 5103-5110.

39 Michels S, Pircher M, Geitzenauer W, Simader C, Götzinger E, Findl $\mathrm{O}$ et al. Value of polarisation-sensitive optical coherence tomography in diseases affecting the retinal pigment epithelium. $\mathrm{Br}$ J Ophthalmol 2008; 92 204-209.

40 Zawadzki RJ, Cense B, Zhang Y, Choi SS, Miller DT, Werner JS. Ultrahigh-resolution optical coherence tomography with monochromatic and chromatic aberration correction. Opt Express 2008; 16: 8126-8143. 